\title{
Neisseria gonorrhoeae isolated at St. Mary's Hospital London, 1980-91
}

\author{
A M Renton, C A Ison, L Whitaker, K Kirtland, E Kupek, J R W Harris
}

\begin{abstract}
Objective-To describe and discuss the trends in the isolation of Neisseria gonorrhoeae from patients attending the Genitourinary Medicine Clinic at St. Mary's Hospital, Paddington, London between 1980 and 1991.

Design-A retrospective study of the total number of gonococci isolated over an eleven year period was performed. In addition, for the years 1988-1991 the number of isolates from homosexual men was analysed by age of the patient, site of infection and HIV antibody status of the patient.
\end{abstract}

Results-The total number of $\boldsymbol{N}$. gonorrhoeae isolates identified declined markedly between 1980 and 1989 from 3670 to 750 isolates. Over the same time period the number of specimens screened for $\boldsymbol{N}$. gonorrhoeae fell by $50 \%$. In 1990 there was an increase in $\boldsymbol{N}$. gonorrhoeae isolates but this was not maintained, and in 1991 the number of $N$. gonorrhoeae fell to its lowest level of 638 isolates. The decrease since 1980 occurred in both men and women although the number of rectal isolates from men showed a steeper decline reaching its lowest level of 24 isolates in 1988. The number of rectal isolates from homosexual men has since increased with a peak in 1990. Many of the infections among homosexual men occurred in older men and included insignificant number of patients who were HIV positive.

Conclusion-Gonorrhoea among attenders at St. Mary's Hospital has declined dramatically since 1980 following trends reported from much of Europe. The increase in gonococcal isolates since 1989 and the peak in 1990 are unexplained but are coincident with a higher number of isolates from homosexual men.

(Genitourin Med 1993;69:286-289)

\section{Introduction}

There has been increasing interest in the epidemiology of post-pubertal gonococcal infection due to the possibility of using gonorrhoea as a marker for sexual activity. ${ }^{1-3}$ The incidence of gonococcal infection has been considered to be an indicator of underlying sexual behaviour in certain populations which may be affected by public health measures to control HIV infection. Also the ability to discriminate between isolates of $N$. gonorrhoeae using typing methods has the potential to provide useful information on the microepidemiology of the disease and the structure of sexual networks. ${ }^{4-5}$

The publication of the UK Government's White Paper in July 1992 gives further emphasis to the renewed interest. ${ }^{6}$ HIV/AIDS and sexual health have been identified as a key area within the national strategy for improving the health of the nation. A reduction in the incidence of gonorrhoea has been set both as an indicator of reduction in the potential for HIV transmission within the population and in order to lessen the incidence of long term sequelae of gonococcal infection. The philosophy of the White Paper is to match targets with a strategy through which they might be achieved, including public awareness campaigns, community based initiatives and education in schools.

The decline in gonorrhoea through the 1980 s has been interpreted as an indicator for the success of education programmes and a change in sexual practices. ${ }^{78}$ More recently the increase in rectal gonorrhoea during 1990 has been taken as evidence of a return to unsafe sexual practices among homosexual men. ${ }^{9-13}$ While this interpretation has been questioned $^{14-16}$ there is evidence for continued transmission of HIV infection among homosexual men in the United Kingdom. ${ }^{17}$

In this paper we review the trends in the number of $N$. gonorrhoeae isolated from patients attending the Genitourinary Medicine (GUM) Clinic at St. Mary's Hospital, London between 1980 and 1991. We have also performed a more detailed analysis of male homosexual patients from whom $N$. gonorrhoeae was isolated between 1988-91 to provide information on the increase in 1990 and any implications this may have for local control measures.

\section{Methods}

Isolation of $N$. gonorrhoeae

Specimens were taken using a disposable loop (urethral and cervical) or a cotton tipped swab (rectal and pharyngeal) and inoculated directly on to GC selective agar containing GC agar base $(36 \mathrm{~g} / \mathrm{l}, \mathrm{BBL})$ supplemented with $1 \%$ IsoVitaleX, vancomycin, colistin, trimethoprim and amphotericin as described previously. ${ }^{18}$ The agar plates were kept in $7 \%$ carbon dioxide at $36^{\circ} \mathrm{C}$ until transported to the laboratory where they were reincubated 


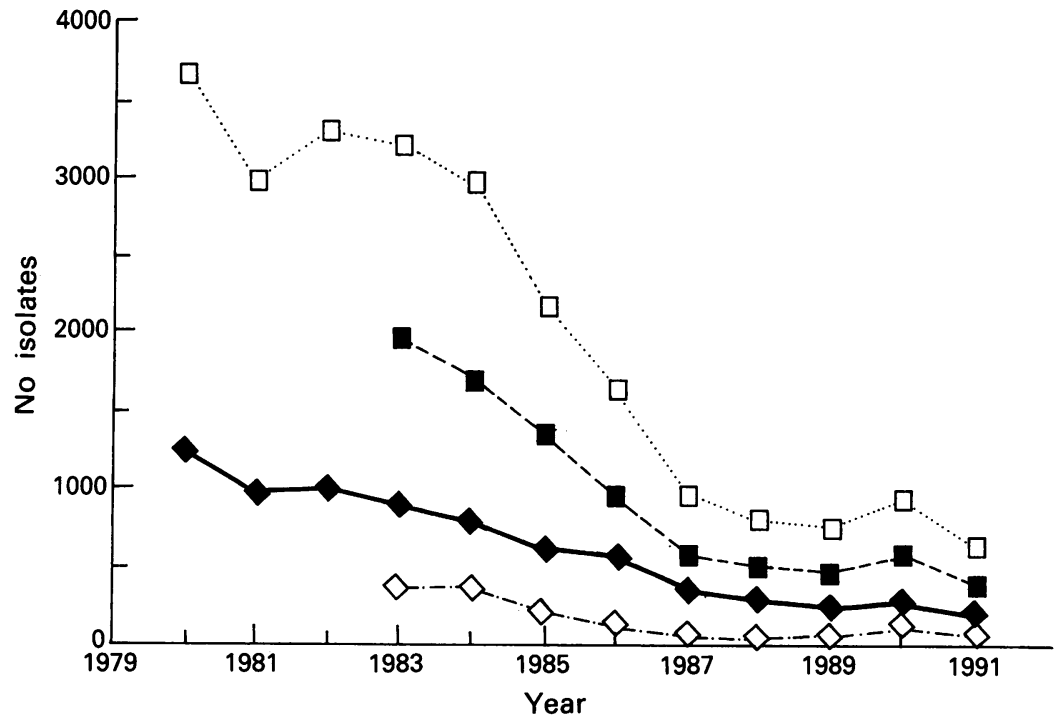

Figure 1 Trends in the isolation of $N$. gonorrhoeae from patients attending St. Mary's Hospital Paddington 1980-91 - - - - - total isolates, - -.-- female isolates, - - - - male urethral isolates and - - $-_{-}--$male rectal isolates. gonorrhoeae. All isolates over the period 1980 to 1991 were included in the analysis.

\section{Patient data}

All patients from whom $N$. gonorrhoeae was saved and typed between January 1988 and December 1991 were identified by hospital number. For those seen at the GUM clinic, notes were sought and found in $97 \%$ of cases. Details of age and HIV test status were recorded from the notes for each visit at which the organism was isolated. Sexual orientation was defined as heterosexual, homosexual or bisexual according to the sex of the partners with whom the patient had had intercourse during the month prior to presentation. Patient based data were then linked to isolates for the purposes of the analysis. Between 1988-91 only 32 bisexual men presented with gonorrhoea which represents $<2 \%$ of the total. In view of the small numbers encountered bisexual men have not been included either alone or among the homosexual group for the analysis presented.

for at least 48 hours before discarded as negative. Typical colonies that were oxidase positive and Gram negative cocci were confirmed as $N$. gonorrhoeae using immunofluorescence (Syva Microtrak Neisseria confirmation test) or carbohydrate utilisation (Quadferm, API Laboratories). Isolates of $N$. gonorrhoeae from consecutive patients attending the GUM clinic between January 1988 and December 1991 were subcultured onto GC agar supplemented with $1 \%$ IsoVitaleX without the addition of antibiotics. The growth was then suspended into $15 \%(\mathrm{v} / \mathrm{v})$ glycerol broth and stored in the vapour phase of liquid nitrogen $\left(-130^{\circ} \mathrm{C}\right)$ for subsequent serotyping ${ }^{19}$ and auxotyping. ${ }^{20}$ The isolates collected and stored were $94 \%$ of the total $N$. gonorrhoeae isolated in $1988,90 \%$ in $1989,99.5 \%$ in 1990 and $99 \cdot 2 \%$ in 1991 .

\section{Collection of specimens}

Specimens were routinely taken from the urethra, cervix and rectum of all women and the urethra of all heterosexual men attending the clinic. The pharynx was sampled in women and heterosexual men if they were contacts of a known case of gonorrhoea or gave a history of practising oral sex. Specimens were taken from the urethra and pharynx of all homosexual men and from the rectum if any history of anal sex was given.

\section{Number of isolates}

The data presented concerning isolation of $N$. gonorrhoeae were prepared in the following manner. For women, urethral and cervical isolates from one patient on the same day were considered as a single isolate. Rectal isolates from women were considered as a distinct isolate even if $N$. gonorrhoeae was also isolated from the urethra and/or cervix. For men, isolates of $N$. gonorrhoeae from the urethra and the rectum were both counted as distinct isolates even if both sites grew $N$.

\section{Results}

Specimens screened for $N$. gonorrhoeae. The total number of specimens screened for $N$. gonorrhoeae fell from 51542 in 1983 to 24180 in 1991 with no major change in sampling policy. Figures for 1980-82 are not available. In 1990, 26956 specimens were screened-a $21 \%$ increase over numbers screened in 1989 (22 271).

Trends in isolation of $N$. gonorrhoeae: 1980-1991

The total number of isolates of $N$. gonorrhoeae obtained in each year from 1980 to 1991 is shown in fig 1 . The number of isolates from women is also shown. The number of isolates from men is shown by site of infection, the urethra and rectum. The number of isolates from the pharynx was $<1 \%$ in each year and has not been shown.

There was a steady decline in the number of $N$. gonorrhoeae isolated from 1980 (3670 isolates) and by 1989 (750 isolates) the number of isolates from both men and women had fallen to approximately $20 \%$ of the numbers isolated in 1980. This trend was reversed briefly in 1990 (940 isolates) when the number of isolates from both sexes increased over 1989 levels. In 1991, the number of isolates fell to the lowest level (638 isolates) since 1980 and represented a $34 \%$ and $30 \%$ reduction over 1990 levels in the number of isolates from women and men respectively.

Urethral isolates from men, both heterosexual and homosexual, followed a similar pattern to the total population. However, the number of rectal isolates, which in men are invariably from homosexuals, decreased more quickly with only 24 such isolates found in 1988. There was an increase in rectal isolates in 1989 (42 isolates) and 1990 (106). While the numbers of rectal isolates fell in 1991 to 61 isolates, this was still the highest level since 1986 . 


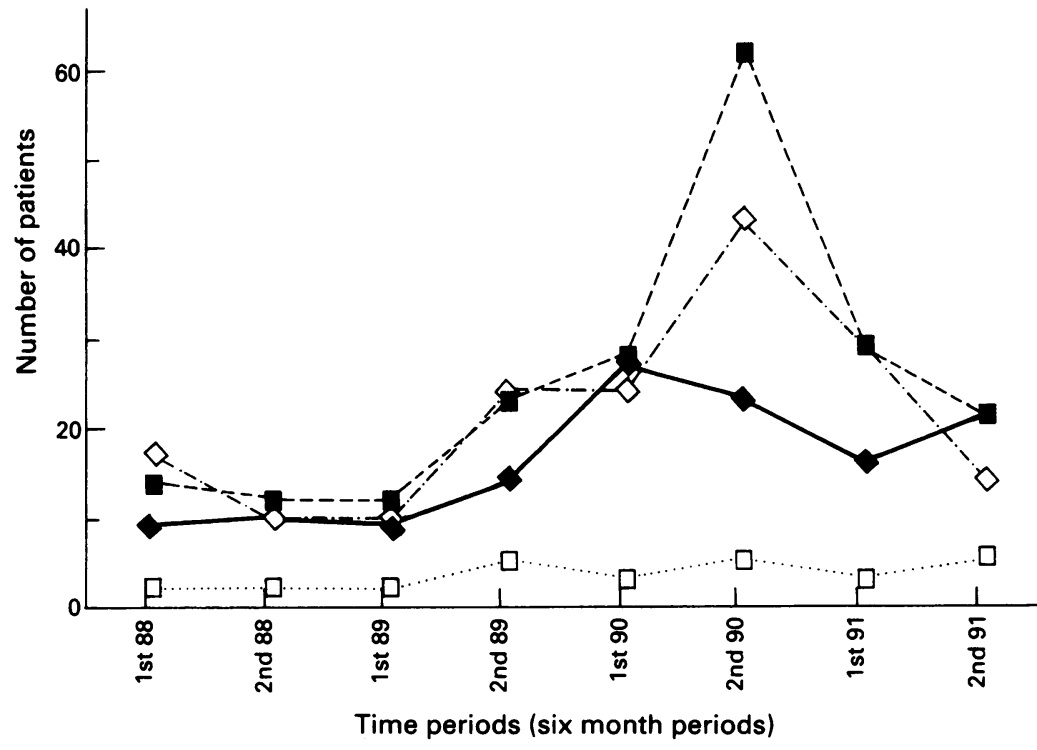

Figure 2 Number of male homosexual patients infected with $N$. gonorrhoeae between

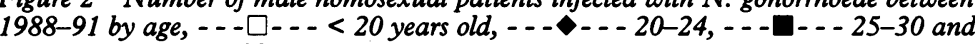
- $\diamond . .-30$ years old.
Gonorrhoea in homosexual men: 1988-91

The combined database obtained by linking the information derived from laboratory and clinical records allowed a more detailed analysis of gonorrhoea in homosexual men attending the GUM clinic between 1988 and 1991. The number of homosexual men from whom $N$. gonorrhoeae was isolated in consecutive six month periods from January 1988 in the age groups $<20$ years old, $20-24,25-30$ and $>30$ is shown in fig 2. N. gonorrhoeae was isolated from homosexual men in all four age groups over the time period studied. There was an increase in the second half of 1990 and the majority of these infections were found in men over the age of 25 years. Isolates were obtained from both the urethra

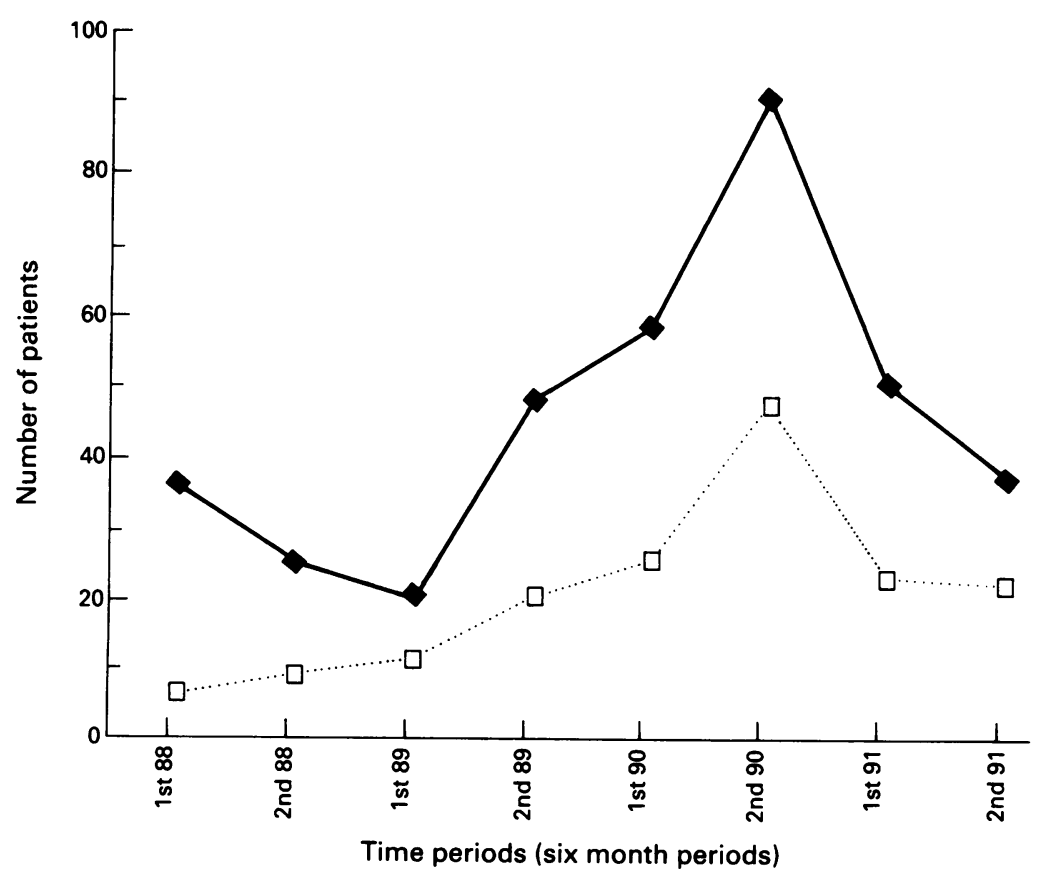

Figure 3 Site of isolation of $N$. gonorrhoeae in male homosexual patients between 1988-91, - - - - - rectum and - - - - urethra. and rectum (fig 3). N. gonorrhoeae was isolated from the pharynx of only four homosexual men during the time period. Of men presenting with rectal infection between 1988-91, the clinical records show that $72 \%$ reported having receptive anal penetration with one or more partner, both casual $(48 \%)$ and regular (47\%), during the preceding month. Of those who had had receptive intercourse with a casual partner, $25 \%$ reported using a condom at some time during that month.

\section{Gonorrhoea in HIV antibody positive homosexual men: 1988-91}

The number of known HIV antibody positive homosexual men who attended the GUM clinic, from whom $N$. gonorrhoeae was isolated, was also examined. Between 1988 and 199117 such men attended with rectal gonorrhoea; $2,6,8$ and 2 of these presented in each successive year (one patient attended twice). Urethral gonorrhoea was seen in 28 HIV positive men distributed more evenly over the time period. These numbers represent $9 \%$ of all attendances with rectal infection and $8 \%$ of all attendances with urethral infection by homosexual men over the four year period.

\section{Discussion}

The total number of isolates of $N$. gonorrhoeae from patients attending the GUM clinic showed a steady decline between 1980 and 1989. There was a small increase in 1990 followed by a decrease in 1991 which was associated with a similar trend in the number of specimens screened. The fall in the isolation of $N$. gonorrhoeae was seen among both men and women. However, isolation from the male rectum, taken as an indicator of homosexual activity, differed slightly from the total population. The initial decrease to 1989 was greater and there was a more marked increase in 1990 and subsequently the number of isolates declined less than from other sites.

The fall in the number of rectal isolates of $N$. gonorrhoeae from men attending our GUM clinic was dramatic. In 1988 we isolated less than $10 \%$ of the gonococci isolated in the years before 1984. This decline has been well documented by others and attributed largely to a fear of acquiring HIV infection and a subsequent change to safer sexual practices. ${ }^{721}$ In addition the decline was taken as evidence for the success of educational campaigns for HIV undertaken by the Government at that time. ${ }^{8}$

The increase in the number of homosexual men attending with $N$. gonorrhoeae during 1990 both at our clinic and elsewhere is largely unexplained. One possible explanation is that younger men who have become sexually active only recently fail to adopt safe sex practices. ${ }^{15}$ However, our data show that during 1990 most cases occurred among homosexual men over the age of 25 years. The number of cases within different age-groups is small and should be interpreted with caution 
but a continued focus of strategies for the promotion of safer sex on both older and younger men may be appropriate. Alternatively the increase in 1990 may reflect natural variation in the number of specimens and isolates of $N$. gonorrhoeae.

We have recently questioned the view that changes in rates of rectal gonorrhoea provide an adequate marker for parallel changes in the frequency of sexual acts that might permit the transmission of HIV. ${ }^{14} 16$ In particular the possibility that gonococcal infection may be transmitted from the pharynx to the rectum should be considered. However, the hypothesis that a substantial proportion of rectal infections identified in this clinic are acquired in this way is not lent support by the very small number of pharyngeal isolates which we have obtained from homosexual men over recent years. We, therefore, believe that the continued isolation of $N$. gonorrhoeae from the rectum and the high proportion of the infected patients who reported receptive anal penetration over the period 1988-91 may suggest that types of sexual contact which permit HIV transmission continue to be practiced. The occurrence of $N$. gonorrhoeae infection in a significant number of homosexual men who were aware of their HIV antibody positive serostatus points to a potential for continued HIV transmission between these men and their sex partners.

Pharyngeal gonorrhoea has a low prevalence in our clinic. This is an unusual finding but could be indicative of the sexual practices of our patient population or of sampling technique and isolation procedures. Over a 15 year period we have been unable to find technical reasons for the lack of pharyngeal isolates and can only presume it relates to our patient population.

Post-pubertal gonorrhoea has been proposed as a measure of levels of sexual activity within a population and hence as an indicator of the potential for HIV transmission. This is based on the assumption of a reliable relationship between incidence of gonorrhoea and sexual activity. This relationship may be less clear than it appears. Changes in incidence may be influenced by the infectivity of individual strains of $N$. gonorrhoeae and their ability to colonise certain patient groups preferentially. Random importation of new strains into populations with steady levels of sexual activity will also affect the incidence. The effectiveness of treatment and contact tracing policies have a further and important influence. Where changes in incidence do reflect modified behaviour, they may be disproportionately influenced by the behaviour of a relatively small number of highly sexually active individuals. In addition the number of isolates from clinic attenders, which is often used as a surrogate for incidence, may be substantially influenced by changes in patterns of clinic attendance and diagnostic testing policy.

Our ability to infer trends in sexual behaviour from trends in STD occurrence will be enhanced by improving the type and quality of information captured in surveillance systems. This can be achieved by improving our knowledge of sexual networks and those elements of sexual behaviour which are important in defining the risk of acquiring a sexually transmitted disease. It is important that all these factors are considered by those responsible for implementing the Health of the Nation strategy.

1 Renton AM, Whitaker L. Using the classical STDs to monitor AIDS prevention. In: Paccaud F, Vader JP, Gutwiller F. eds Assessing AIDS Prevention. Basel. Birkhauser Verlag, 1992:32-51.

2 Renton AM, Whitaker L. Using STD occurrence to monitor AIDS prevention. A report to the European Community. Cahiers de recherche et documentation. Community. Cahiers de

3 Catchpole MA. Sexually transmitted diseases in England and Wales 1981-90. Communicable Disease Report Review 1992;2:R1-7.

4 Ross JD, McMillan A, Young H. Changing trends of gonococcal infection in homosexual men in Edinburgh Epidemiol Infect 1991;107:585-90.

5 Ison CA, Whitaker L, Renton AM. Concordance of auxotype/serovar classes of Neisseria gonorrhoeae between sexual contacts. Epidemiol Infect 1992;109:265-71.

6 Secretary of State for Health. The Health of the nation: a Strategy for Health in England. London, HMSO, 1992 (CM 1986).

7 Gellan MCA, Ison CA. Declining incidence of gonorrhoea in London: a response to fear of AIDS? Lancet 1986;ii:920.

8 Weller IVD, Hindley DJ, Adler MW, Meldrum JT. Gonorrhoea in homosexual men and media coverage of the acquired immune deficiency syndrome in London $B M F$ 1984;289:1041.

9 French PD, Mercey DE, Tomlinson DR, Harris JRW Preventing the spread of HIV infection. $B M \mathcal{F}^{1991}$; 302:962.

10 Singaratnam AE, Boag F, Barton SE, Hawkins DA Lawrence AG. Preventing the spread of HIV infection $B M F$ 1991;302:469.

11 van den Hoek JAR, van Griensven GJP, Coutinho RA. Increase in unsafe homosexual behaviour. Lancet 1990; 336:179-80.

12 Waugh MA. Resurgent gonorrhoea in homosexual men. Lancet 1991;337:375.

13 Riley VC. Resurgent gonorrhoea in homosexual men. Lancet 1991;337:183.

14 Tomlinson DR, French PD, Harris JRW, Mercey DE Does rectal gonorrhoea reflect unsafe sex? Lancet 1991 337:501-2.

15 Renton AM, Whitaker L. Preventing the spread of HIV infection. $B M \mathcal{F}$ 1991;302:1207-8.

16 Whitaker L, Renton AM. A theoretical problem of interpreting the recently reported increase in homosexual gonorrhoea. Eur $\mathcal{F}$ Epid 1992;8:187-91.

17 Waight PA, Miller E. Incidence of HIV infection among homosexual men. BMF 1991;303:311.

18 Ison CA, Gedney G, Easmon CSF. Chromosomal resistance of gonococci to antibiotics. Genitourin Med 1987; 63:239-43.

19 Knapp JS, Tam MR, Nowinski RC, Holmes KK, Sandstrom EG. Serological classification of Neisseria gonorrhoeae with use of monoclonal antibodies to gonococcal outer membrane protein Int $\mathcal{F}$ Infect Dis 1984 150:44-8.

20 Copley CG, Egglestone SI. Auxotyping of Neisseria gonorrhoeae isolated in the United Kingdom. $F \mathrm{Med}$ Microbiol. 1983;26:295-302.

21 Judson FN. Fear of AIDS and gonorrhoea rates in homosexual men. Lancet 1983;ii:159-60. 